\title{
El Código de Ética de la Asociación Cubana de Bibliotecarios (ASCUBI) en el contexto internacional
}

The Code of Ethics of the Cuban Association of Librarians (ASCUBI) in the international context

\section{Emilio SETIÉn QUESADA}

Secretario de Trabajo Profesional. Asociación Cubana de Bibliotecarios. esetien@infomed.sld.cu

\section{Resumen}

Se presentan los antecedentes del código actual de la Asociación y se comentan los contenidos que lo insertan en lo más generalizado de este tipo de documentos en lo relacionado con la protección a la confidencialidad, el mantenimiento de un alto nivel profesional, la prioridad de los intereses de los lectores, la garantía al acceso a los documentos y sus contenidos sin restricciones, proporcionando una información completa, objetiva e imparcial.

Palabras clave: Ética de la Información. Códigos éticos. Asociación Cubana de Bibliotecarios. Estudios comparativos

\section{Introducción}

Son los colegios y asociaciones profesionales, amparados por la ley y con personalidad propia, las entidades que se encargan de reglamentar los principios éticos del ejercicio de las profesiones mediante la elaboración de los códigos de ética, denominados también como códigos deontológicos.

A diferencia de las regulaciones legislativas establecidas para las profesiones por los órganos estatales de un país, los códigos de ética son elaborados por los propios profesionales de las distintas esferas. El incumplimiento de las normas establecidas en ellos no implican sanciones de carácter legal. Son sanciones de carácter moral como amonestaciones, o separaciones del colectivo asociado.

El primer Código de ética para los bibliotecarios cubanos fue elaborado por la entonces denominada Asociación Nacional de Profesionales de la Biblioteca, a principios de la década del 50 del siglo pasado. Fue publicado en el primer número de la revista Cuba Bibliotecológica, órgano oficial de la Asociación (1). Como todos los códigos de este tipo, el documento estaba

\begin{abstract}
The background of the current ethical code of the Cuban Association of Librarians is discussed, and its different aspects are analyzed with a focus on its connections with other relevant international regulations: the protection of confidentiality, maintaining high professional standards, giving priority to the readers' interests, the right of access to documents and their contents without restrictions, and the provision of complete, objective and impartial information.
\end{abstract}

Keywords: Information ethics. Codes of ethics. Cuban Association of Librarians. Comparative Studies.

conformado por un conjunto de reglas a seguir en el desempeño profesional y era de obligatorio cumplimiento por parte de los miembros de la Asociación.

No es hasta el segundo congreso de la actual Asociación Cubana de Bibliotecarios, celebrado en su nueva etapa iniciada en 1985 , que se aprueba un nuevo código de ética, sustentado por la declaración de principios del propio Congreso (2).

El código entonces aprobado reconocía, entre otros, que los bibliotecarios cubanos estaban conscientes de la importancia de su papel en el desarrollo de la vida social del país, como informadores y formadores de cultura y de valores ciudadanos, por lo que ofrecían servicios bibliotecarios de excelencia, manteniendo una actualización profesional permanente y estimulando la de sus colegas, propiciando la participación en eventos que redundaran en el enriquecimiento del desarrollo profesional y en el conocimiento y reconocimiento de la Bibliotecología Cubana.

Establecía, asimismo, la separación de los intereses particulares de los institucionales y la obligación de no derivar relaciones ni beneficios económicos personales del trabajo con los lec- 
tores, como medio de lucro o para obtener prebendas en su propio beneficio.

En general los códigos de ética no pueden verse como reglas y normas de conducta inmutables, independientes del desarrollo histórico. No pueden considerarse válidos para toda época, para todo lugar, para todo grupo social. Como reflejo de la conciencia social están sustentados por los principios que predominan en cada agrupación humana histórico concreta.

No obstante, los códigos de ética profesional, independientemente de las necesarias diferencias que les imprimen las razones antes apuntadas, presentan determinados rasgos que responden a la esencia de cada profesión en cualquiera latitud, aunque varíen con el tiempo.

Merced a esos rasgos esenciales es posible analizar la inserción del código de ética de ASCUBI en el conjunto de los que se establecen para la profesión en otros países.

\section{El código de ética de ASCUBI en la actualidad}

Hoy, de cara al III Congreso de ASCUBI, y como parte de su proceso orgánico, las asambleas de las filiales provinciales analizan una nueva versión ampliada del código. Esta versión tiene como peculiaridad su vinculación con el Regla- mento establecido por el Sistema de Bibliotecas Públicas del país, en el que se asumen para el personal del sistema los deberes y derechos definidos por el Código de la Asociación.

En el estudio realizado por el profesor Luis Bermello, titulado Análisis comparativo de 12 códigos de ética bibliotecaria (3): se observa que los contenidos más reiterados en los códigos consultados por el autor son

- La protección a la confidencialidad de, los lectores y de los documentos consultados

- El mantenimiento de un alto nivel profesional mediante la superación

- La prioridad de los intereses de los lectores, colegas y otros ante los privados

- La garantía al acceso a los documentos y sus contenidos sin restricciones, proporcionando una información completa, objetiva e imparcial

A continuación se presenta un análisis del código de ASCUBI que se encuentra hoy en proceso de aprobación, a la luz de lo que sucede en los países estudiados por el profesor Bermello. En ese estudio se puede observar que los contenidos de los códigos varían en extensión. De 22 rubros identificados, algunos países sólo incluyen 3 y otros llegan a contemplar 16.

\begin{tabular}{|c|c|c|c|c|c|c|c|c|c|c|c|c|c|}
\hline & & ca & us & it & $g b$ & pt & $d k$ & au & $n z$ & $m x$ & $\mathrm{cl}$ & jp & $h k$ \\
\hline & Obligaciones Con Los Usuarios & & & & & & & & & & & & \\
\hline 1 & Las obligaciones con los usuarios son su deber primario & & & & $x$ & $x$ & $x$ & & $x$ & & & $x$ & $x$ \\
\hline 2 & Garantizar el acceso a la información pública sin restricciones & $\mathrm{x}$ & & $x$ & $\mathrm{x}$ & $\mathrm{x}$ & $\mathrm{x}$ & $\mathrm{x}$ & & $x$ & & $\mathrm{x}$ & $\mathrm{x}$ \\
\hline 3 & Proporcionar información completa, objetiva, imparcial & & $x$ & $\mathrm{x}$ & & $x$ & $x$ & $\mathrm{x}$ & & $\mathrm{x}$ & $x$ & $\mathrm{x}$ & $\mathrm{x}$ \\
\hline 4 & $\begin{array}{l}\text { Las convicciones personales no deben interferir con sus } \\
\text { obligaciones }\end{array}$ & & $x$ & $x$ & & $x$ & & $x$ & & $x$ & & & $x$ \\
\hline 5 & No se discriminará al usuario (sexo, color de la piel, etc. & & & $x$ & $\mathrm{x}$ & & & $\mathrm{x}$ & $\mathrm{x}$ & $\mathrm{x}$ & & $\mathrm{x}$ & $\mathrm{x}$ \\
\hline 6 & Oposición a las políticas de censura & & $x$ & $x$ & & $x$ & & $x$ & & $\mathrm{x}$ & & $\mathrm{x}$ & $x$ \\
\hline 7 & $\begin{array}{l}\text { Proteger la confidencialidad de los usuarios y de la información } \\
\text { consultada }\end{array}$ & $x$ & $x$ & $x$ & $x$ & $x$ & $x$ & $x$ & $x$ & $x$ & $x$ & $x$ & $x$ \\
\hline 8 & $\begin{array}{l}\begin{array}{l}\text { No anteponer los intereses privadosa los de los usuarios, colegas, } \\
\text { etc. }\end{array} \\
\end{array}$ & & $x$ & $x$ & $x$ & $x$ & & $x$ & $x$ & $x$ & $x$ & $x$ & $x$ \\
\hline 9 & Respaldar las libertades intelectuales / valores democráticos & $\mathrm{x}$ & $\mathrm{x}$ & $\mathrm{x}$ & & $\mathrm{x}$ & $\mathrm{x}$ & & & & & $\mathrm{x}$ & \\
\hline \multirow[t]{2}{*}{10} & Trato correcto y cortés a los usuarios & & $\mathrm{x}$ & & & & $\mathrm{x}$ & & & $\mathrm{x}$ & & & \\
\hline & Obligaciones Con La Profesion & & & & & & & & & & & & \\
\hline 11 & Honrar la profesión & & & $\mathrm{x}$ & $\mathrm{x}$ & & & & $\mathrm{x}$ & $\mathrm{x}$ & $\mathrm{x}$ & & $\mathrm{x}$ \\
\hline 12 & $\begin{array}{l}\text { Mantener un alto nivel profesional (excelencia) mediante la } \\
\text { superación }\end{array}$ & & $x$ & $x$ & $x$ & $x$ & $x$ & $x$ & $x$ & $\mathrm{x}$ & $x$ & $\mathrm{x}$ & $x$ \\
\hline 13 & Relaciones con los colegas basadas en el respeto y la colaboracion & & $\mathrm{x}$ & $x$ & & $\mathrm{x}$ & & $\mathrm{x}$ & & $\mathrm{x}$ & $\mathrm{x}$ & $\mathrm{x}$ & \\
\hline 14 & Proporcionar servicios de alta calidad & $x$ & $\mathrm{x}$ & $\mathrm{x}$ & & $\mathrm{x}$ & $\mathrm{x}$ & $x$ & & & & & \\
\hline 15 & Representar adecuadamente a la institución empleadora & & $\mathrm{x}$ & & $\mathrm{x}$ & & & & & $\mathrm{x}$ & $\mathrm{x}$ & & \\
\hline
\end{tabular}




\begin{tabular}{|c|c|c|c|c|c|c|c|c|c|c|}
\hline 16 & Luchar por condiciones de trabajo decorosas & $x$ & & & & $\mathrm{x}$ & & $\mathrm{x}$ & $x$ & \\
\hline & Obligaciones Con La Información & & & & & & & & & \\
\hline 17 & Promover la preservación de la información & & $\mathrm{x}$ & & & & $\mathrm{x}$ & & $\mathrm{x}$ & \\
\hline 18 & Garantizar la trasmisión del conocimiento & & $x$ & & & & $\mathrm{x}$ & $\mathrm{x}$ & & \\
\hline 19 & Promover el flujo de información e ideas & & $x$ & $\mathrm{x}$ & & & $x$ & & & $\mathrm{x}$ \\
\hline 20 & Desarrollar colecciones apropiadas y organizadas & $x$ & $x$ & & $x$ & $x$ & $x$ & $x$ & $x$ & $x$ \\
\hline 21 & Formular políticas sobre los servicios bibliotecarios & & & & $x$ & & $x$ & $x$ & & \\
\hline 22 & Respetar los derechos de la propiedad intelectual & $x$ & & & & $\mathrm{x}$ & & $\mathrm{x}$ & & \\
\hline
\end{tabular}

Tabla I. Aspectos contemplados en los códigos de ética bibliotecaria

La versión actual del código de ASCUBI tiene propuesta la estructura siguiente

- Lineamientos Generales

- Principios Generales del Código de Ética

- De las relaciones con los lectores y con los servicios

- De las relaciones con los colegas y con la profesión
- Deberes del bibliotecario

- Derechos del bibliotecario

- Disposiciones finales

Evidencia correspondencias con los códigos estudiados por Bermello, expresadas en contenidos que se agrupan de la forma siguiente.

\begin{tabular}{|c|c|c|}
\hline $\begin{array}{c}\text { En los códigos consultados por } \\
\text { Bermello }\end{array}$ & & En el código actual de ASCUBI \\
\hline $\begin{array}{c}\text { Rubros sobre obligaciones con los } \\
\text { lectores }\end{array}$ & & $\begin{array}{c}\text { Rubros sobre relaciones con los } \\
\text { lectores }\end{array}$ \\
\hline \multicolumn{3}{|c|}{ Protección a la confidencialidad } \\
\hline La profesión & & Deberes del bibiotecario \\
\hline & $\begin{array}{l}\text { Mantenimientos de un alto nivel profesional } \\
\text { prioridad de los intereses de los lectores, ante } \\
\text { los privados }\end{array}$ & \\
\hline \multirow[t]{2}{*}{ El acceso a los contenidos } & & Los servicios \\
\hline & $\begin{array}{c}\text { Garantía para el acceso a los documentos y } \\
\text { sus contenidos sin restricciones, } \\
\text { proporcionando una información completa, } \\
\text { objetiva e imparcial. }\end{array}$ & \\
\hline
\end{tabular}

Tabla II. Correspondencias entre los códigos

Con respecto a la protección a la confidencialidad de los lectores y de los documentos consultados, el código de ASCUBI en proceso de aprobación se pronuncia en sus principios generales por la privacidad y confidencialidad declarando que el bibliotecario tiene la obligación de guardar la privacidad y confidencialidad de todos los lectores, preservando el secreto profesional de datos personales, búsquedas y cualquier uso de la información.

Sobre el mantenimiento de un alto nivel profesional mediante la superación, también en los principios generales se puede leer que la disposición constante a la superación continua, el intercambio profesional, el trabajo en equipo y en red y la calidad de su quehacer es lo que define el profesionalismo de un bibliotecario. $Y$ en los derechos del bibliotecario el código establece que el profesional ha de tener acceso a las acciones de superación y a la literatura profesional, así como al intercambio con sus colegas.

En lo referente a la prioridad de los intereses de los lectores, colegas y otros ante los privados, los lineamientos generales del Código reconocen, en primera instancia, los valores sociales sin la interferencia o imposición de valores personales. En este sentido define las obligaciones del bibliotecario en cuatro dimensiones:

- Obligaciones sociales, pues participa, como agente de una institución social, no sólo en 
los objetivos de su institución, sino en los de la sociedad en su conjunto.

- Obligaciones con el entorno, que incluyen, fundamentalmente, las entendidas con las asociaciones profesionales, con los usuarios o lectores y con el sistema bibliotecario.

- Obligaciones en el ejercicio de su trabajo dirigidas hacia la organización institucional, los superiores y los colegas.

- Obligaciones hacia uno mismo en la preservación de su propia vida, en el cuidado de su familia y en la defensa de sus opiniones en el contexto profesional.

Y en su sección de deberes establece que el bibliotecario no podrá derivar relaciones ni beneficios económicos personales de su trabajo con los lectores, ni valerse del cargo que desempeñe para obtener ventajas de cualquier índole.

A la garantía para el acceso a los documentos y sus contenidos sin restricciones, proporcionando una información completa, objetiva e imparcial, el código contiene varios pronunciamientos en sus principios generales, a saber:

- De la libertad intelectual: Todos los lectores tienen acceso a las bibliotecas sea cual sea su condición, raza o lengua, preservando la memoria colectiva y en defensa de la herencia cultural de nuestro pueblo.

- De la Propiedad intelectual: La actuación profesional del bibliotecario descansa en el reconocimiento de las normativas legales sobre la propiedad intelectual y el derecho de autor que permiten establecer los límites en el uso de la información sin entrar en conflictos de intereses.

- De las Colecciones amplias y objetivas: El bibliotecario es el formador del patrimonio bibliográfico de las naciones por lo que no debe ejercer censura ni criterios personales en la selección de documentos y colecciones de bibliotecas. Su actuación estará en correspondencia con la demanda universal de la información y con los objetivos, normas y regulaciones de la institución correspondiente.

En la sección de deberes: establece, además, que la información que brinda el bibliotecario debe estar organizada y ser lo más completa posible, satisfaciendo las necesidades y el acceso a la información de los lectores, sin distinción de raza, sexo, credo, edad, o cualquier otra condición social.

En resumen, aparte de las peculiaridades que presenta el código de ética de la Asociación
Cubana de Bibliotecarios -fundamentadas en los principios aprobados desde el II Congreso de la Asociación-, el documento responde a la esencia de la profesión y a las preocupaciones más comunes del sector, con enfoque similar al del resto de los países incluídos en este análisis. Como otros códigos de ética profesional, las sanciones que se contemplan en él no tienen carácter legal. Son sólo sanciones morales.

\section{Notas}

Cuba Bibliotecológic. Op. cit. en bibliografía.

ASCUBI. Op. cit. en bibliografía.

Bermello, Luis. Op. cit. en bibliografía.

Bermello. op. cit.

\section{Bibliografía}

Aznar, Hugo. Ética y periodismo. Barcelona, Paidós, 1999.

ASCUBI. http://www.cultstgo.cult.cu/biblioteca/ascubi.htm

Bermello, Luis. Análisis comparativo de 12 códigos de ética bibliotecaria. CD ROM

Congreso Internacional De Información Info 2004. Memorias: Habana, Cuba del 12-16 de abril del 2004 info 95, info 97, info 99, Info 2002, info 2004. Palacio de las Convenciones de La Habana

Cuba Bibliotecológica. 1(1):19-20,1953

FAIFE. <http://www.faife.dk/>

Recibido: 24-08-2010.

Aceptado: $24-08-2010$ 